\title{
Identification of potential novel biomarkers for abdominal aortic aneurysm based on comprehensive analysis of circRNA-miRNA-mRNA networks
}

\author{
TAN LI $^{1^{*}}$, TIANLONG WANG $^{2 *}$, LIRONG YAN $^{3^{*}}$ and CHUNYAN MA ${ }^{1}$ \\ ${ }^{1}$ Department of Cardiovascular Ultrasound, ${ }^{2}$ The First Clinical College of China Medical University and \\ ${ }^{3}$ Tumor Etiology and Screening Department of Cancer Institute and General Surgery, \\ The First Hospital of China Medical University, Shenyang, Liaoning 110001, P.R. China
}

Received June 24, 2021; Accepted October 8, 2021

DOI: 10.3892/etm.2021.10903

\begin{abstract}
Abdominal aortic aneurysm (AAA) is a life-threatening disorder and, therefore, investigation into its underlying mechanisms in light of the competing endogenous RNAs (ceRNAs) hypothesis has gradually increased. However, there is still lacking systematic analysis on AAA-associated circular RNA (circRNA)-microRNA (miRNA/miR)-messenger RNA (mRNA) interaction networks based on bioinformatics methods. The present study attempted to identify novel molecular biomarkers for AAA by profiling circRNA-miRNA-mRNA networks using three public microarray datasets (GSE7084, GSE57691 and GSE144431). A total of 135 differentially expressed genes (DEGs) and 142 differentially expressed circRNAs were detected using the limma $\mathrm{R}$ package with the statistical threshold of $\mathrm{P}<0.05$ and $\log _{2}$ fold change $(\mathrm{FC}) \mid>1.5$.
\end{abstract}

Correspondence to: Dr Chunyan Ma, Department of Cardiovascular Ultrasound, The First Hospital of China Medical University, 155 Nanjing Bei Street, Shenyang, Liaoning 110001, P.R. China

E-mail: cmu1h_mcy@126.com

*Contributed equally

Abbreviations: AAA, abdominal aortic aneurysm; VSMC, vascular smooth muscle cell; miRNAs, microRNAs; lncRNAs, long non-coding RNAs; circRNAs, circular RNAs; mRNA, messenger RNA; ceRNAs, competing endogenous RNAs; RT-qPCR, reverse transcription-quantitative PCR; GEO, gene expression omnibus; DEGs, differentially expressed genes; DECs, differentially expressed circRNAs; MCODE, molecular complex detection; GO, Gene Ontology; KEGG, Kyoto Encyclopedia of Genes and Genomes; BP, biological processes; CC, cellular component; $\mathrm{MF}$, molecular function; ROC, receiver operating characteristic; $\mathrm{CNN} 1$, calponin 1; AUC, area under the curve; CEMIP, cell migration inducing hyaluronan-binding protein; SOST, sclerostin

Key words: abdominal aortic aneurysm, competing endogenous RNA, circular RNA, microRNA, bioinformatics
In addition, 12 circRNA-miRNA-mRNA axes were identified to construct upregulated and downregulated ceRNA networks using Cytoscape. Based on molecular complex detection algorithm, (hsa_circ_0057691/0092108/0006845/0082182)miR-330-5p-calponin 1 (CNN1) and (hsa_circ_0061482/00 11450/0008351/0004121)-miR-326-CD8a molecule (CD8A) were recognized as the center axes in ceRNA networks. Reverse transcription-quantitative PCR results verified the significant downregulation of CNN1 and upregulation of CD8A in human AAA tissues $(\mathrm{P}<0.05)$. In addition, four upregulated circRNA/mRNA axes, and five downregulated circRNA/mRNA axes were revealed to have possible biological functions in the pathogenesis of AAA using the Cytoscape software. Receiver operating characteristic analysis demonstrated the accuracy of these nine DEGs involved in these axes for AAA diagnosis with area under the curves $>0.80$. The present study revealed novel circRNA-miRNA-mRNA networks associated with AAA, especially for CNN1 and CD8A axes with the potential function of 'focal adhesion' and 'immune response', respectively. Overall, the present findings may provide evidence to explore the implicated ceRNAs in the molecular mechanisms and as novel biomarkers for AAA.

\section{Introduction}

Abdominal aortic aneurysm (AAA) is a degenerative vascular disease caused by the progressive weakening and dilation of abdominal aorta with a diameter $>3-\mathrm{cm}$ (1). As a complex and serious vascular surgery disease, AAA has an extremely high mortality rate, especially in populations aged $>65$ years (2). Immune inflammatory response, extracellular matrix degradation and vascular smooth muscle cell (VSMC) dysfunction are suggested to be important factors in AAA formation (3). However, the exact regulatory mechanisms underlying AAA remain unclear. In addition, more studies and experiments are still needed to explore how to prevent AAA formation and progression earlier.

Non-coding RNA (ncRNA) is composed of diverse RNA transcripts including microRNA (miRNA), long non-coding RNA (lncRNA) and circular RNA (circRNA), a number of which are known to act as transcriptional regulators (4). 
miRNAs that are 21-23 nucleotides in length can specifically bind with messenger RNAs (mRNAs) and reversely regulate the expression of genes (5). By contrast, lncRNAs and circRNAs can function as competing endogenous RNAs (ceRNAs) and sponge miRNAs, thus offsetting the negative regulation of miRNAs on target mRNAs (6). The role of the ceRNA network is extensive and has been observed in the regulation of disease progression, including cardiovascular problems (7). This builds a notable basis for exploring pathogenetic mechanisms of AAA through constructing a ceRNA network mediated via IncRNAs or circRNAs.

In fact, to the best of our knowledge, the majority of experimental studies mainly focus on the effects of IncRNA-miRNA-mRNA axes on AAA risk (8-12). The evolution of novel computational tools is a rapidly growing field of interest, which provides putative predictions of ceRNA interactions (7). Based on bioinformatics analysis, Tian et al (13) constructed lncRNA-miRNA-mRNA networks for AAA to reveal six novel IncRNA-miRNA-mRNA axes (five upregulated and one downregulated) closely associated with the pathogenesis of AAA. Current evidence has confirmed that circRNAs may have effects on AAA development through modulating endothelial cells, macrophages and VSMCs (14). Zhao et al (15) reported that circRNA cerebellar degeneration-related protein 1 antisense RNA is downregulated in AAA and is associated with the upregulation of miR-7, resulting in the low-degree expression of cytoskeleton-associated protein 4 and dysregulation of VSMCs. Yue et al (16) identified that circRNA core-binding factor subunit $\beta$ is a sponge of miR-28-5p that is highly expressed in AAA and that induces the downregulation of glutamate ionotropic receptor AMPA type subunit 4 and LY6/PLAUR domain-containing 3, both of which are linked with VSMC apoptosis. However, data on circRNAs in human AAA are limited, and there is still a lack of systematic analysis on circRNA-miRNA-mRNA interaction networks in AAA based on bioinformatics analysis.

The current bioinformatics study aimed to establish AAA-associated circRNA-miRNA-mRNA networks and to identify the center ceRNA axes. Subsequently, the expression levels of mRNAs involved in center axes were verified by reverse transcription-quantitative PCR (RT-qPCR). In addition, the potential biological functions of circRNA-miRNA-mRNA axes that are closely linked with AAA were explored and the clinical significance of key ceRNA-associated mRNAs was evaluated. The results of the present study may aid the improved understanding of AAA pathogenesis, especially in terms of ceRNA implication; it may also provide some potential molecular targets and novel ideas for further experiments to explore novel therapeutic approaches for AAA.

\section{Materials and methods}

Microarray datasets. First, appropriate public datasets of human AAA were collected by using Gene Expression Omnibus (GEO) database in National Center of Biotechnology Information (http://www.ncbi.nlm.nih.gov/geo/). Overall, three microarray datasets (GSE7084, GSE57691 and GSE144431) were included in the present study (Table SI) (17-19). In GSE7084, seven AAA samples and eight healthy samples were involved for mRNA expression profiles by GPL2507 platform. In GSE57691, 49 AAA samples and 10 healthy samples were included for mRNA expression profiles using GPL10558 platform. In GSE144431, four AAA samples and four healthy samples were involved for circRNA expression profiles by GPL21825 platform. Principal component analysis (PCA) was utilized to reduce the dimension and assess the data availability of aforementioned datasets (20). The PCA score plots of dimension (Dim) 1 and 2 were visualized using $\mathrm{R}$ studio (version 4.0.3) (21). Superior group differentiation is demonstrated by higher Dim scores, indicating the availability of datasets.

Differential expression analysis. Steps of differential expression analysis of each dataset are presented below. The differentially expressed genes (DEGs) and differentially expressed circRNAs (DECs) were detected by applying the limma R package (version 3.46.0; https://bioconductor. org/packages/limma/) with the statistical threshold of $\mathrm{P}<0.05$ and $\mid \log _{2}$ fold change (FC) $>1.5$ in R studio (version 4.0.3) (21). The DEGs and DECs were annotated according to the corresponding platform information, respectively. Pheatmap R package (version 1.0.12; https://cran.r-project.org/web/packages/pheatmap/index.html) was used to generate heatmaps in R studio (version 4.0.3) (21). DEGs in GSE7084 and GSE57691 or DECs in GSE144431 have been annotated as official symbols already. However, due to the different sequencing chips used, GSE7084 and GSE57691 may have different official symbols for the same DEGs. Therefore, the public database for Annotation, Visualization and Integrated Discovery Online tool (DAVID; version 6.8; https://david. ncifcrf.gov/) was used to convert the DEGs of GSE7084 and GSE57691 into uniform Entrez_Gene_ID (22). In this way, the Entrez_Gene_ID of DEGs could be overlapped between GSE7084 and GSE57691 to obtain the common up and downregulated DEGs. However, this process was not necessary for GSE144431 since GSE144431 only contained information for DECs, which cannot be overlapped with DEG datasets.

Construction of circRNA-miRNA-mRNA networks and center axis exploration. Subsequently, online tools were used to predict the potential target miRNAs of DEGs and DECs, respectively. The miRNA-circRNA pairs were predicted through the CircInteractome database (version 1.0) (https://circinteractome.nia.nih.gov/index.html) (23). The miRWalk database (version 2.0) (http://mirwalk. umm.uni-heidelberg.de/) (24) and TargetScan database (version 7.2) (http://www.targetscan.org) were used to predict miRNA-mRNA pairs (25). Furthermore, miRNA-circRNA and miRNA-mRNA pairs were overlapped to select common target miRNAs of DEGs and DECs. Finally, upregulated circRNAs/mRNAs and downregulated circRNAs/mRNAs were selected to construct two different ceRNA networks, respectively. Cytoscape software (version 3.6.1) (26) was used for the visualization of ceRNA networks. The molecular complex detection (MCODE) method (a plugin in Cytoscape; version 2.0.0) was performed to identify highly interconnected clusters in ceRNA networks (27), and the circRNA-miRNA-mRNA axes involved in the clusters were considered as center axes. The criterion for MCODE analysis was set up as default parameters. 
Patient sample collection and RT-qPCR validation. RT-qPCR validation was conducted on DEGs in the center ceRNA axes. A total of four patients with AAA had large and non-ruptured cases and full-thickness aneurysmal abdominal aortic tissues were collected from them during the open surgical repair, whereas four control samples were derived from non-aneurysmal abdominal aortas of organ donors during kidney transplantation. The study was approved by the Ethics Committee of the First Hospital of China Medical University (approval no. 2020-146-2; Shenyang, China) and was carried out in accordance with the Declaration of Helsinki (28). Written informed consent was obtained from each patient. Characteristics of AAA and control subjects are presented in Table SII. All patients with AAA were males with ages of $68.0 \pm 7.8$ years, whereas control individuals had the age distribution of $58.3 \pm 3.9$ years, with and one female and three males. Sample collection took place in the First Hospital of China Medical University between July 2020 and April 2021. Patients with AAA were diagnosed using CT angiography and underwent open surgery. Individuals with congenital disorders, malignant tumors, hematological diseases, autoimmune diseases, severe organ failure or previous aortic surgery were excluded.

Whole RNA in samples was extracted from tissues using RNAiso Plus (Takara Bio, Inc.). The gDNA Eraser with strong DNA decomposition activity can remove genomic DNA at $42^{\circ} \mathrm{C}$ for $2 \mathrm{~min}$ and the samples treated by gDNA Eraser can be directly synthesized into cDNA by reverse transcription reaction at $37^{\circ} \mathrm{C}$ for 15 min using PrimeScript ${ }^{\mathrm{TM}} \mathrm{RT}$ Reagent kit (Takara Bio, Inc.). RT-qPCR was performed with the SYBR ${ }^{\circledR}$ Premix Ex Taq ${ }^{\mathrm{TM}}$ II (Takara Bio, Inc.) according to the manufacturer's protocol. RT-qPCR thermocycling conditions included: Initial denaturation step at $95^{\circ} \mathrm{C}$ for $2 \mathrm{~min}$, followed by 40 cycles of $10 \mathrm{sec}$ at $95^{\circ} \mathrm{C}, 20 \mathrm{sec}$ at $58^{\circ} \mathrm{C}$ and $30 \mathrm{sec}$ at $72^{\circ} \mathrm{C}$. $\beta$-actin was used as the reference gene to calculate relative fold difference using $2^{-\Delta \Delta C q}$ method (29). Primer sequences for CNN1, CD8a molecule (CD8A) and $\beta$-actin are presented in Table SIII. Gene-specific primers were designed with Primer Express Software 3.0 (Applied Biosystems; Thermo Fisher Scientific, Inc.) and purchased from Beijing Genomics Institute (https://www.genomics.cn/).

Statistical analysis. Quantitative data are expressed as means \pm standard deviation and were analyzed using SPSS 17.0 statistical software (SPSS, Inc.). The unpaired t-test was applied for comparisons between AAA and control groups. $\mathrm{P}<0.05$ was considered to indicate a statistically significant difference.

Functional and pathway enrichment for DEGs. The results of Kyoto Encyclopedia of Genes and Genomes (KEGG) pathways (https://www.genome.jp/kegg/) and Gene Ontology (GO) terms (https://geneontology.org/) including biological processes (BP), cellular component (CC) and molecular function (MF) enrichment analyses for common DEGs were obtained using the DAVID online tool (version 6.8; https://david.ncifcrf.gov/) (22). The threshold was $\mathrm{P}<0.05$. GOplot R package (version 1.0.2) (30) was used for the visualization of the enrichment analysis results and $\mathrm{z}$-score was introduced to evaluate the results.

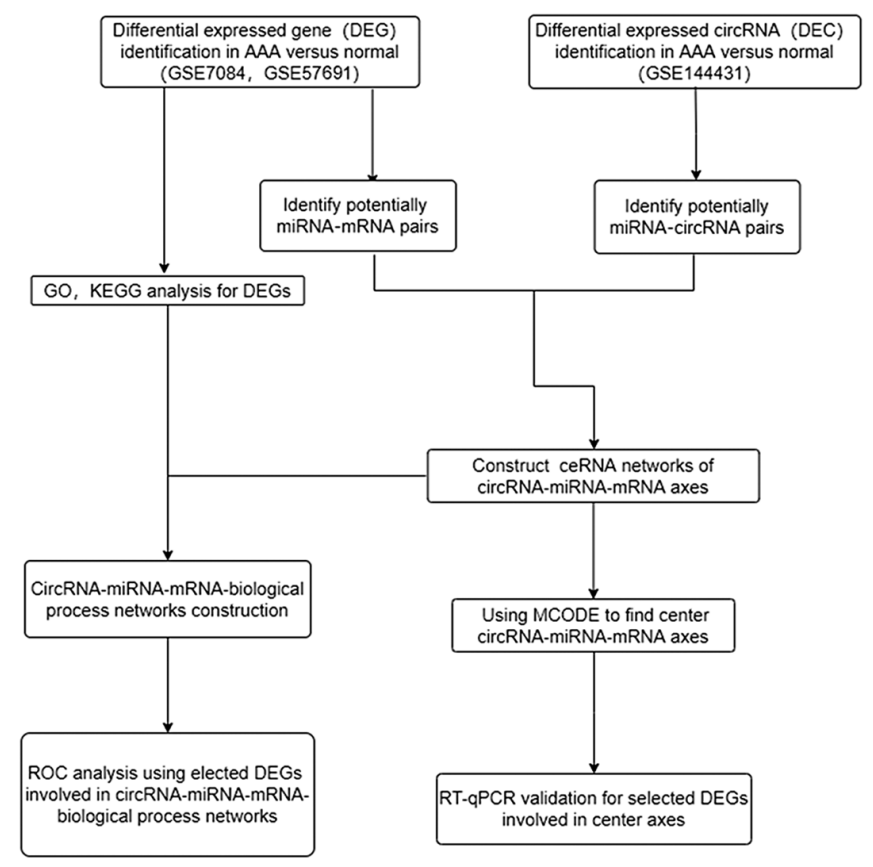

Figure 1. Workflow for the present study. DEGs, differentially expressed genes; AAA, abdominal aortic aneurysm; circRNA, circular RNA; miRNA, microRNA; mRNA, messenger RNA; GO, Gene Ontology; KEGG, Kyoto Encyclopedia of Genes and Genomes; ceRNA, competing endogenous RNA; RT-qPCR, reverse transcription-quantitative; ROC, receiver operating characteristic.

Construction of circRNA-miRNA-mRNA-biological function networks. Based on the previous GO analysis, DEG-GO terms pairs and circRNA-miRNA-mRNA-biological function networks were constructed using Cytoscape software (version 3.6.1) (26). Similarly, upregulated circRNA/mRNA and downregulated circRNAs/mRNAs were selected to construct two separate networks.

Receiver operating characteristic (ROC) analysis. To further explore the clinical diagnostic value of circRNA-miRNA-mRNA-biological process network-associated DEGs, ROC analysis was conducted through ROC R package (version 1.68.1; https://bioconductor. org/packages/release/bioc/html/ROC.html) in R studio (version 4.0.3) (21). The data were derived from gene mRNA expression levels in each sample of GSE7084 and GSE57691 with a further normalization.

\section{Results}

Identification of DEGs and DECs. The workflow of the present study is presented in Fig. 1. PCA confirmed a clear separation between AAA and healthy groups of three datasets (Fig. S1A). Fig. S1B indicates the 643 DEGs, 454 DEGs and 142 DECs in GSE7084, GSE57691 and GSE144431, respectively. Matrix metallopeptidase 9 (MMP9) is the most upregulated DEG and protein phosphatase 1 regulatory subunit 3C (PPP1R3C) is the most downregulated DEG in the heatmap of GSE7084 (Fig. S1C). For the heatmap of GSE57691, Ig kappa chain V-III region HAH-like (LOC642113) is the most upregulated DEG and integrin subunit alpha 8 (ITGA8) is the most 
A

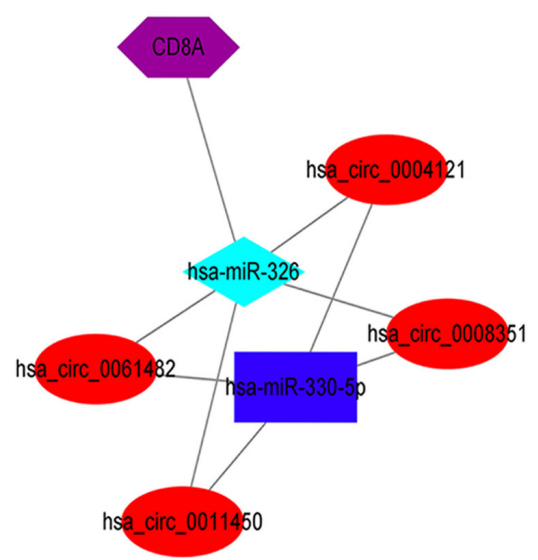

B

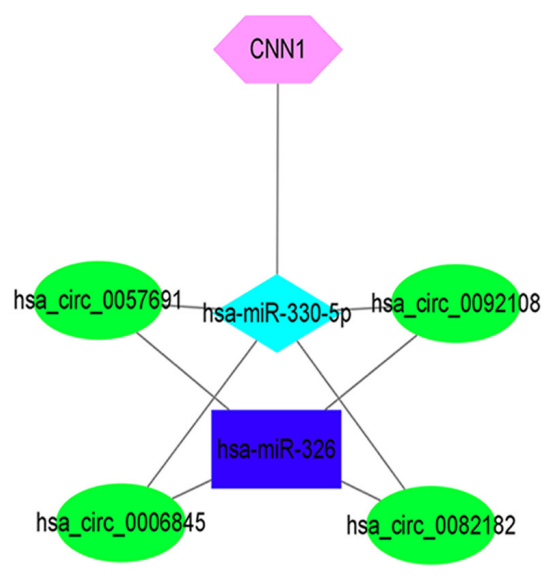

Figure 2. MCODE network clustering analysis based on GSE7084, GSE57691 and GSE144431. (A) The upregulated circRNAs/mRNAs. (B) The downregulated circRNAs/mRNAs. Red ellipse, upregulated circRNAs; purple hexagon, upregulated mRNAs; green ellipse, downregulated circRNAs; pink hexagon, downregulated mRNAs; light blue diamond, common target miRNAs of circRNAs and mRNAs; dark blue rectangle, predicted miRNAs of circRNAs or mRNAs. CNN1, calponin 1; miR, microRNA.

A

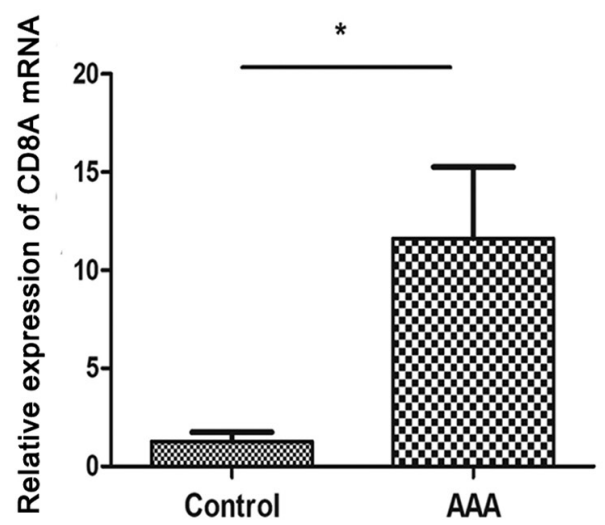

B

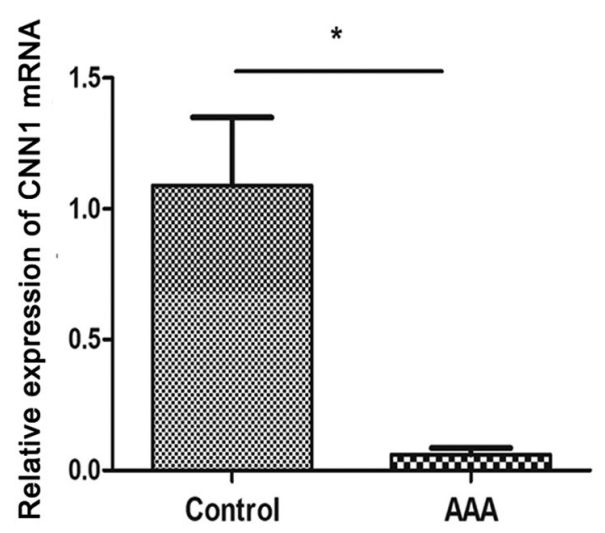

Figure 3. Validation of CNN1 and CD8A by reverse transcription-quantitative PCR based on the collected aortic samples from patients in the present study. Relative expression of (A) CD8A and (B) CNN1 mRNA in control and AAA tissues. "P<0.05. CNN1, calponin 1.

downregulated DEG (Fig. S1D). In the heatmap of GSE144431, hsa_circ_0001588 represents the most upregulated DEC and hsa_circ_0092291 represents the most downregulated DEC (Fig. S1E). Tables SIV and SV present a total of 643 DEGs and 454 DEGs in GSE7084 and GSE57691, respectively. Finally, a total of 135 common DEGs (62 upregulated and 73 downregulated) between GSE7084 and GSE57691 (Fig. S1F and Table SVI) and 142 DECs (64 upregulated and 78 downregulated) in GSE144431 (Table SVII) were identified for the construction of ceRNA networks.

Construction of ceRNA regulatory networks in AAA. To explore the effect of DECs on DEGs mediated by binding with miRNAs, upregulated and downregulated ceRNA networks were constructed. As presented in Fig. S2, the upregulated ceRNA networks contained four circRNA-miRNA-mRNA axes [CD8A,IL-10 receptor subunit $\alpha$ (IL10RA), semaphorin 4A (SEMA4A) and cell migration inducing hyaluronan-binding protein (CEMIP)] and downregulated ceRNA networks contained eight circRNA-miRNA-mRNA axes [atonal bHLH transcription factor 8 (ATOH8), prune homolog 2 with $\mathrm{BCH}$ domain (PRUNE2), calponin 1 (CNN1), zinc finger and BTB domain-containing 16 (ZBTB16), sclerostin (SOST), transmembrane protein 47 (TMEM47), F-box protein 32 (FBXO32) and nuclear factor IA (NFIA)]. In addition, Fig. S3 displays a total of 12 circRNA-miRNA-mRNA networks constructed based on every single DEG.

Identification of center ceRNA axes and RT-qPCR validation. Based on MCODE algorithm, (hsa_circ_0057691/0092108/00 06845/0082182)-miR-330-5p-CNN1 and (hsa_circ_0061482/ 0011450/0008351/0004121)-miR-326-CD8A were regarded as two center axes (Fig. 2). RT-qPCR analysis further displayed that patients with AAA had significantly lower expression of CNN1 and higher expression of CD8A compared with controls $(\mathrm{P}<0.05$; Fig. 3$)$, which was consistent with the results of microarray analysis.

Functional prediction of DEGs in AAA. To explore the potential biological function characteristics of detected 
A

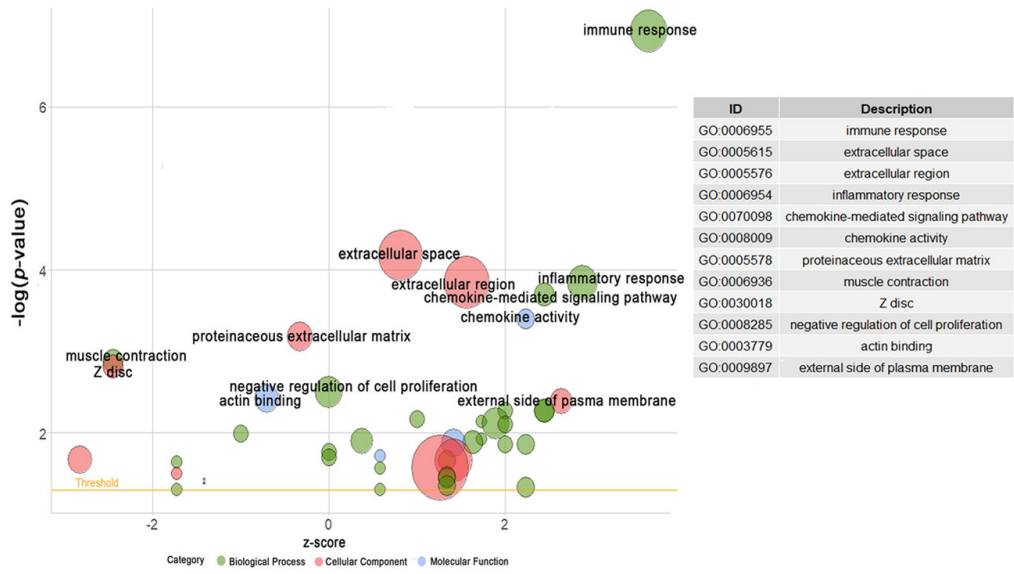

B

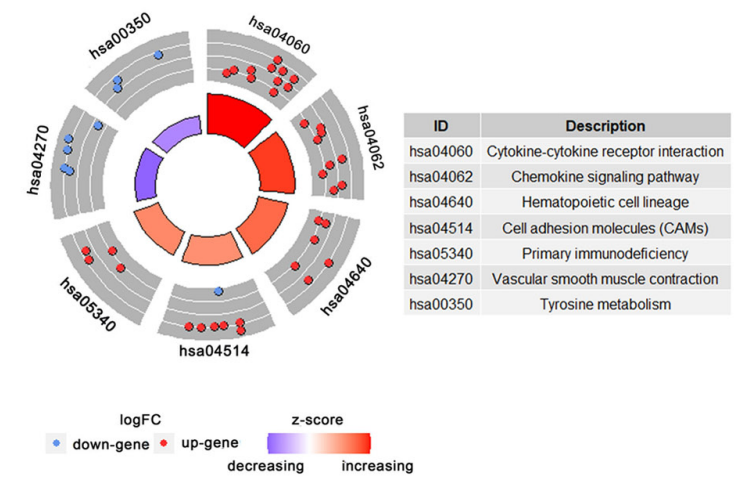

Figure 4. Visualization of GO and KEGG enrichment analysis results for DEGs. (A) Bubble plot for twelve important GO terms. (B) Circular plot for seven important KEGG pathways. The inner circle displays the z-score of the pathway. Outer circle displays the number of up and downregulated DEGs in the pathway. GO, Gene Ontology; KEGG, Kyoto Encyclopedia of Genes and Genomes; DEGs, differentially expressed genes.

A

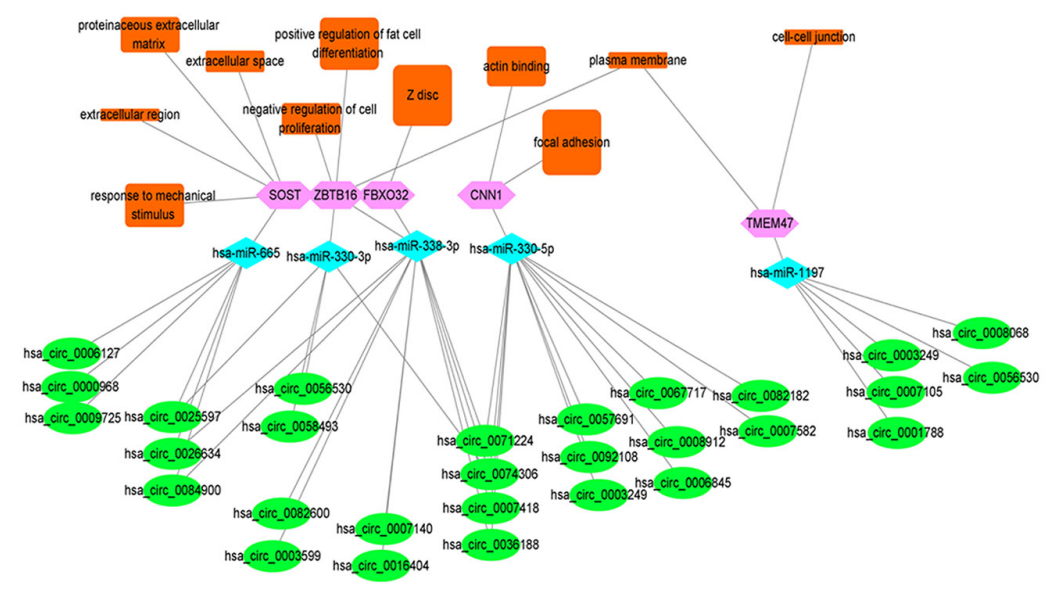

B

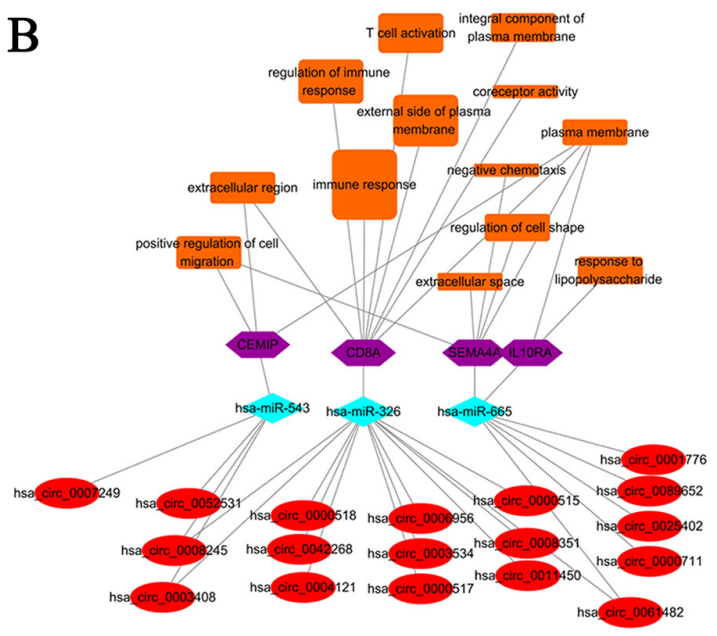

Figure 5. Construction of circRNA-miRNA-mRNA biological function networks. (A) Networks for five downregulated axes. (B) Networks for four upregulated axes. Green ellipse, downregulated circRNAs; Pink hexagon, downregulated mRNAs; Red ellipse, upregulated circRNAs; Purple hexagon, upregulated mRNAs; light blue diamond, common target miRNAs of circRNAs and mRNAs; Orange rectangle, GO terms. The larger the rectangle, the more positive or negative z-score for GO terms in upregulated or downregulated axes. circRNA, circular RNA; miRNA/miR, microRNA; mRNA, messenger RNA; CNN1, calponin 1; SOST, sclerostin; TMEM47, transmembrane protein 47; ZBTB16, zinc finger and BTB domain-containing 16; FBXO32, F-box protein 32; CEMIP, cell migration inducing hyaluronan-binding protein; IL10RA, interleukin 10 receptor subunit $\alpha$; SEMA4A, semaphorin 4A; CD8A, CD8a molecule.

common DEGs, GO terms and KEGG pathway analyses were conducted. A total of 45 GO terms and 11 KEGG pathways were obtained. GO analysis suggested that DEGs were mainly involved in 'immune response' and 'inflammatory response' of $\mathrm{BP}$, 'extracellular region' and ' $\mathrm{Z}$ disc' of $\mathrm{CC}$ and 'chemokine activity' and 'actin binding' of MF. KEGG pathways indicated that DEGs were associated with 'cytokine-cytokine receptor interaction', 'chemokine signaling pathway' and 'vascular smooth muscle contraction'. A total of 12 important GO terms and seven crucial KEGG pathways were visualized into Bubble plot and Circular plot, respectively (Fig. 4). All GO terms and KEGG pathways can be obtained in Tables SVIII and SIX. Based on the $\mathrm{z}$-score, GO terms such as 'immune response', 'inflammatory response' and 'chemokine activity' were upregulated and 'muscle contraction', 'Z disc' and 'actin binding' were downregulated. And KEGG pathways such as 'cytokine-cytokine receptor interaction' and 'chemokine signaling pathway' were upregulated, while 'vascular smooth muscle contraction' and 'tyrosine metabolism' were downregulated.

The potential biological functions of ceRNA axes in $A A A$. By combining GO terms and ceRNA networks, we could identify the potential biological functions of 12 circRNA-miRNA-mRNA axes. Finally, nine axes (four upregulated and five downregulated) were linked with GO terms (Fig. 5). Overall, four upregulated axes (SEMA4A, CEMIP, IL10RA and CD8A) interacted with 13 GO terms, including the 'immune response', 'regulation of immune response', 'T cell activation', 'positive regulation of cell migration', 'extracellular region', 'external side of plasma membrane', 'integral component of plasma membrane', 
Table I. Differentially expressed genes in circular RNA-micro RNA-messenger RNA-biological process networks.

\begin{tabular}{llll}
\hline Gene ID & Gene symbol & \multicolumn{1}{c}{ Gene full name } & Group \\
\hline 1264 & CNN1 & Calponin 1 & Down \\
50964 & SOST & Sclerostin & Down \\
83604 & TMEM47 & Transmembrane protein 47 & Down \\
7704 & ZBTB16 & Zinc finger and BTB domain-containing 16 & Down \\
114907 & FBXO32 & F-box protein 32 & Down \\
57214 & CEMIP & Cell migration inducing hyaluronan-binding protein & Up \\
3587 & IL10RA & Interleukin 10 receptor subunit alpha & Up \\
64218 & SEMA4A & Semaphorin 4A & Up \\
925 & CD8A & CD8a molecule & Up \\
\hline
\end{tabular}

Table II. AUC and 95\% CI for differentially expressed genes in circular RNA-micro RNA-messenger RNA-biological process networks.

\begin{tabular}{lcc}
\hline Gene symbol & AUC & $95 \%$ CI \\
\hline CNN1 & 0.83 & $0.742-0.923$ \\
TMEM47 & 0.93 & $0.877-0.988$ \\
SOST & 0.91 & $0.842-0.982$ \\
ZBTB16 & 0.96 & $0.917-0.998$ \\
FBXO32 & 0.93 & $0.875-0.986$ \\
CEMIP & 0.85 & $0.751-0.942$ \\
IL10RA & 0.93 & $0.865-0.994$ \\
SEMA4A & 0.99 & $0.968-1.000$ \\
CD8A & 0.88 & $0.792-0.964$ \\
\hline
\end{tabular}

AUC, area under the curve; DEGs, differentially expressed genes; CI, confidence interval; CNN1, calponin 1; SOST, sclerostin; TMEM47, transmembrane protein 47; ZBTB16, zinc finger and BTB domain-containing 16; FBXO32, F-box protein 32; CEMIP, cell migration inducing hyaluronan-binding protein; IL10RA, interleukin 10 receptor subunit $\alpha$; SEMA4A, semaphorin 4A; CD8A, CD8a molecule.

'coreceptor activity', 'negative chemotaxis', 'regulation of cell shape', 'extracellular space', 'plasma membrane', 'response to lipopolysaccharide' (Fig. 5B). Furthermore, five downregulated axes (CNN1, ZBTB16, SOST, TMEM47 and FBXO32) interacted with $11 \mathrm{GO}$ terms, including 'focal adhesion', 'Z disc', 'actin binding', 'proteinaceous extracellular matrix', 'negative regulation of cell proliferation', 'extracellular region', 'response to mechanical stimulus', 'extracellular space', 'positive regulation of fat cell differentiation', 'plasma membrane', 'cell-cell junction' (Fig. 5A).

ROC analysis of ceRNA related DEGs. A total of nine DEGs involved in circRNA-miRNA-mRNA-biological function networks were selected for ROC analysis to explore their clinical diagnostic value. Table I presents the basic information for these nine DEGs. The area under the curve (AUC) and 95\% confidence interval for every DEG are listed in Table II, and ROC curves are displayed in Fig. 6. As a result, all selected DEGs had AUCs $>0.80$ with a high sensitivity and specificity.

\section{Discussion}

To the best of our knowledge, few in-depth bioinformatics studies on the key circRNAs of ceRNA networks involved in AAA pathogenesis have been conducted. Although some GEO datasets in the present study have been used to perform other analyses $(2,13,31,32)$, there are no studies exploring circRNA-miRNA-mRNA networks for AAA with these datasets. For the first time, the present study screened crucial circRNA-miRNA-mRNA axes through a series of bioinformatics tools and validated some key molecular targets. The results may deepen the understanding of molecular mechanisms in AAA formation and offer potential candidate biomarkers for AAA.

First, 135 DEGs (62 upregulated and 73 downregulated) and 142 DECs (64 upregulated and 78 downregulated) from GEO database were identified. Then, miRNAs of DEGs and DECs were predicted using online tools and the intersections were revealed. Finally, 12 circRNA-miRNA-mRNA axes were discovered to construct ceRNA networks. Moreover, CNN1 and CD8A associated axes were revealed to be in the top significant modules based on MCODE algorithm. RT-qPCR analysis further verified that human AAA tissues had lower expression of CNN1 and higher expression of CD8A compared with controls. KEGG enrichment analysis revealed that DEGs were mainly enriched in 'cytokine-cytokine receptor interaction', 'chemokine signaling pathway' and 'vascular smooth muscle contraction'. By linking circRNA-miRNA-mRNA axes to the corresponding GO terms, a total of nine axes (CNN1, ZBTB16, SOST, TMEM47, FBXO32, SEMA4A, CEMIP, IL10RA and CD8A) were identified with potential biological functions. Further ROC analyses were conducted on these DEGs, which indicated that they might be potential predictors of AAA with significant diagnostic value. Notably, CNN1 and CD8A related axes were also matched to GO terms, in which CNN1 was associated with 'focal adhesion' and 'actin binding', while CD8A was associated with 'immune response' and ' $T$ cell activation', further suggesting the important role of these two axes in AAA disease. 
A

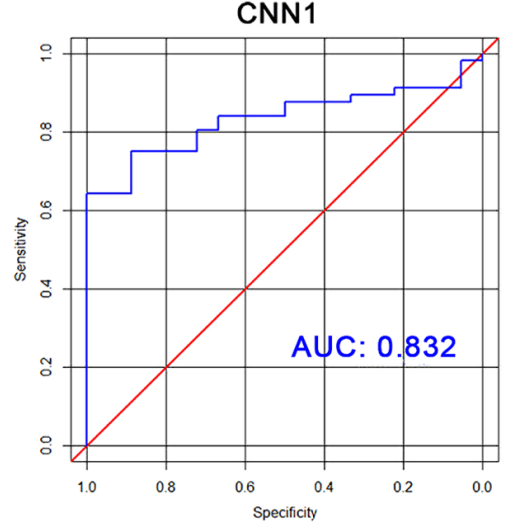

D

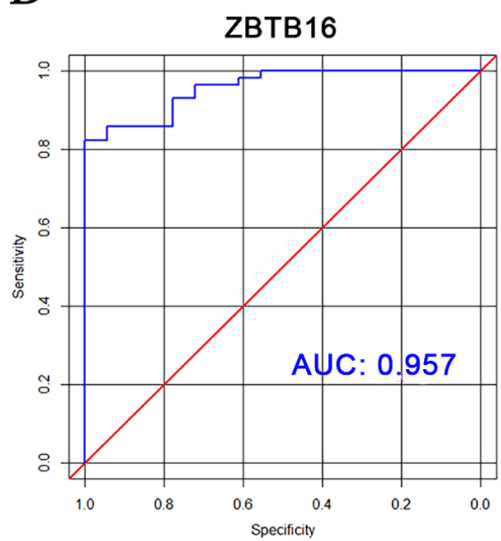

G

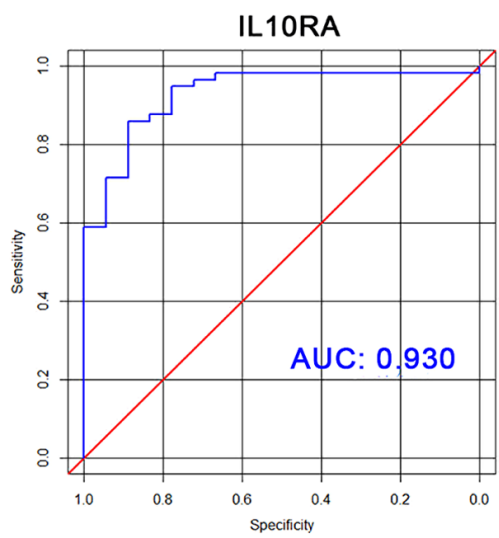

B

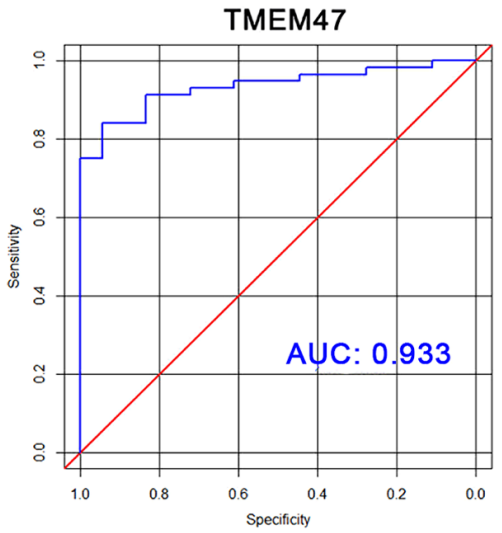

$\mathrm{E}$

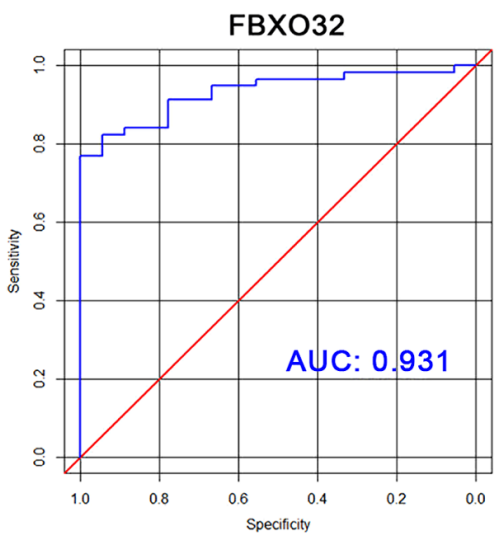

$\mathrm{H}$

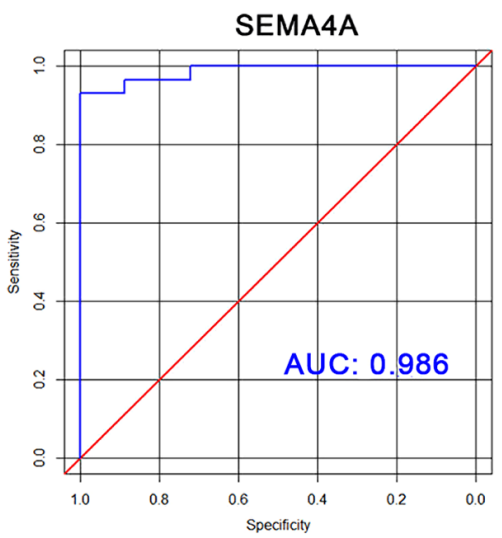

$\mathrm{C}$

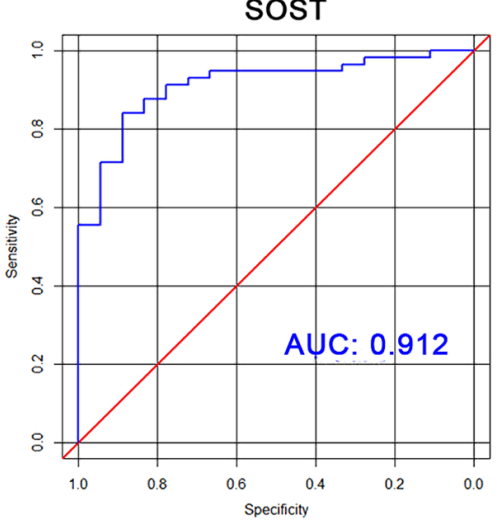

F

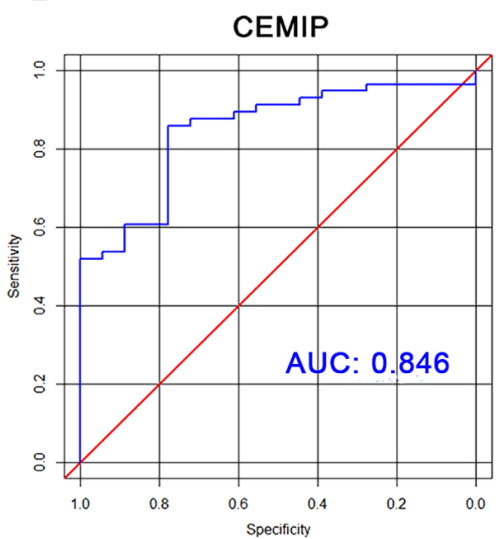

I

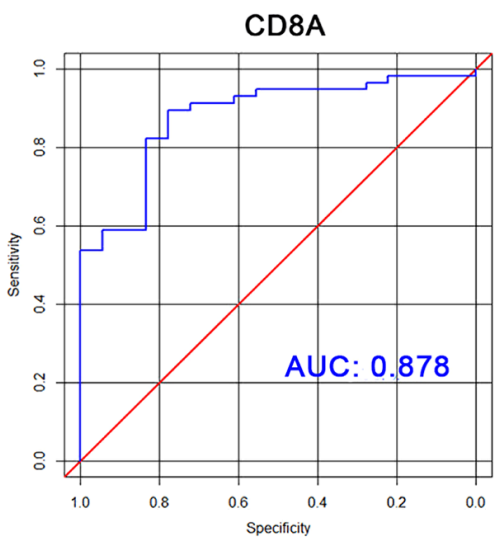

Figure 6. ROC curves for DEGs involved in circRNA-miRNA-mRNA-biological process axes. ROC curves for (A) CNN1, (B) TMEM47, (C) SOST, (D) ZBTB16, (E) FBXO32, (F) CEMIP, (G) IL10RA, (H) SEMA4A and (I) CD8A, respectively. circRNA, circular RNA; miRNA/miR, microRNA; mRNA, messenger RNA; CNN1, calponin 1; SOST, sclerostin; TMEM47, transmembrane protein 47; ZBTB16, zinc finger and BTB domain-containing 16; FBXO32, F-box protein 32; CEMIP, cell migration inducing hyaluronan-binding protein; IL10RA, interleukin 10 receptor subunit $\alpha$; SEMA4A, semaphorin 4A; CD8A, CD8a molecule; ROC, receiver operating characteristic; DEGs, differentially expressed genes.

Calponin is considered as the marker of VSMC differentiation and contractility $(33,34)$. As a notable homologous gene of calponin, CNN1 has been demonstrated to be downregulated in intracranial aneurysm and thoracic aortic aneurysm (35-37). A previous study showed that CNN1 deficiency can lead to decreased contractile force in VSMCs, indicating the loss of elasticity in blood vessel (38). Recently, Lesiak et al (39) found that the number of CNN1-positive cells is reduced in all layers of human AAA. However, there are limited data concerning the role of CNN1 associated ceRNAs in AAA.

Therefore, to the best of our knowledge, the present study was the first to reveal that $\mathrm{CNN} 1$ was downregulated in AAA specimens and targeted by miR-330-5p and four circRNAs (hsa_circ_0057691/0092108/0006845/0082182). The present study provided evidence that $\mathrm{CNN} 1$ could be a key molecule in AAA formation from the perspective of ceRNA theory. As 
for CD8A, its expression can be mainly detected in $\mathrm{CD}^{+}$cytotoxic T cells (40). Previous research revealed that $\mathrm{CD}^{+} \mathrm{T}$ cells can induce the death of VSMCs by secreting various cytotoxic mediators, thus promoting AAA formation (41), whereas CD8 ${ }^{+}$ T cell depletion reduces the development of AAA (42). A bioinformatics study indicated that CD8A expression is inhibited by the overexpression of miR-370-3p, leading to the loss of $\mathrm{CD}^{+}$ $\mathrm{T}$ cell immune function and sepsis occurrence (43). Another previous bioinformatics analysis suggested that CD8A expression is upregulated in large AAA (18). The present research further observed novel circRNA-miRNA pairs (hsa_circ_00 61482/0011450/0008351/0004121-miR-326) targeting CD8A which was highly expressed in AAA tissues.

Except for the CNN1 and CD8A associated axes, there were three upregulated axes (CEMIP, SEMA4A and IL10RA) and four downregulated axes (SOST, ZBTB16, FBXO32 and TMEM47) associated with GO terms, which were significantly enriched in positive regulation of cell migration, extracellular region, plasma membrane, extracellular space, regulation of cell shape, negative chemotaxis/response to lipopolysaccharide, response to mechanical stimulus, extracellular region, proteinaceous extracellular matrix, extracellular space, negative regulation of cell proliferation, positive regulation of fat cell differentiation, $\mathrm{Z}$ disc, plasma membrane and cell-cell junction. Notably, these biological processes are also important processes involved in the pathophysiological mechanisms of AAA. These results indicated that these ceRNA networks may be potential molecular factors involved in AAA pathogenesis. It is of great importance to intervene these pathways during the initial stages to effectively reduce the development of AAA.

For upregulated axes-associated DEGs, CEMIP can regulate the proliferation and migration of VSMCs in atherosclerosis and is associated with the carcinogenesis and progression of cancer $(44,45)$. In intervertebral disc degeneration, circ_001653-silencing can downregulate CEMIP by upregulating miR-486-3p to alleviate nucleus pulposus apoptosis and metabolic imbalance of extracellular matrix (46). SEMA4A is generally regarded as a key gene in positive regulation of immune-cell activation, differentiation and migration (47). As a part of IL10 receptors, IL10RA is associated with anti-inflammatory response and can be inhibited by miR-142-5p to induce inflammatory bowel disease (48). For downregulated axes-associated DEGs, SOST has been discovered to be downregulated in human aortic aneurysm and its overexpression can prevent aortic aneurysm development by inhibiting the Wnt signaling pathway (49). In murine models of ankylosing spondylitis, SOST activation can be suppressed by miR-96 to improve osteoblast differentiation and bone formation (50). ZBTB16 has the potential to enhance mitochondrial respiratory function and promote brown adipocyte changes (51). FBXO32 has been reported to be targeted by various miRNAs (miR-1-3p, miR-29a/b-3p and miR-133a/b-3p) to induce muscle wasting in diabetic murine models (52). TMEM47 can organize the epithelial cell junction maturation and morphogenesis by influencing localization of a subset of tight junction proteins and associated actomyosin structures (53). However, the role of these DEGs and the relevant regulatory networks in AAA occurrence are largely unknown. The present study provided evidence for future studies that concentrate on how circRNA-miRNA-mRNA networks take part in AAA.

The present study had some limitations. First, public datasets for analyzing AAA associated circRNA and miRNA expression profiles were limited. Second, there was a lack of AAA-associated clinical information in the public database. Third, the sample size of the collected AAA and control tissues were small because of the prevalence of endovascular aneurysm repair over open surgical repair, in addition to the difficulty in obtaining healthy aortic samples. This also restricted the further validation of CNN1 and CD8A expression on protein level. In addition, further in vitro and in vivo experiments should be performed to verify the present results, especially the causal regulatory relationships among circRNA, miRNA and mRNA.

In summary, the present study revealed novel circRNA-miRNA-mRNA axes and their potential biological functions implicated in AAA pathogenesis. CNN1 and CD8A associated ceRNA axes were revealed to be center axes, and the expression levels of CNN1 and CD8A were also validated using RT-qPCR. To the best of our knowledge, the present study is the first in-depth bioinformatics study to identify potential novel biomarkers for AAA in light of comprehensive analysis of circRNA-miRNA-mRNA networks; therefore, these findings may provide clues to demonstrate the molecular mechanisms and therapeutic targets for AAA in terms of ceRNA theory.

\section{Acknowledgements}

Not applicable.

\section{Funding}

This study was supported by grants from the National Natural Science Foundation of China (grant nos. 82001828 and 81871373) and Natural Science Foundation of Liaoning Province (grant no. 2020-BS-102).

\section{Availability of data and materials}

The sequencing datasets generated and/or analyzed during the current study are available in the GEO database (https://www. ncbi.nlm.nih.gov/geo/query/acc.cgi?acc=GSE7084; https://www. ncbi.nlm.nih.gov/geo/query/acc.cgi?acc=GSE57691; https:// www.ncbi.nlm.nih.gov/geo/query/acc.cgi?acc=GSE144431). The non-sequencing datasets used and/or analyzed during the current study are available from the corresponding author on reasonable request.

\section{Authors' contributions}

TL designed the research, performed the experiments and wrote the manuscript. TW contributed to drafting the article and performing the bioinformatics analysis. LY participated in the data analysis and interpretation. CM was involved in study conception and revising the manuscript critically for important intellectual content. TL and CM confirm the authenticity of all the raw data. All authors have read and approved the final manuscript. 


\section{Ethics approval and consent to participate}

The study was approved by the Ethics Committee of the First Hospital of China Medical University (approval no. 2020-146-2) and was carried out in accordance with the Declaration of Helsinki. Written informed consent was obtained from each patient.

\section{Patient consent for publication}

Not applicable.

\section{Competing interests}

The authors declare that they have no competing interests.

\section{References}

1. Sakalihasan N, Michel JB, Katsargyris A, Kuivaniemi H, Defraigne JO, Nchimi A, Powell JT, Yoshimura K and Hultgren R: Abdominal aortic aneurysms. Nat Rev Dis Primers 4: 34, 2018.

2. Chen S, Yang D, Lei C, Li Y, Sun X, Chen M, Wu X and Zheng Y: Identification of crucial genes in abdominal aortic aneurysm by WGCNA. PeerJ 7: e7873, 2019.

3. Wu QY, Cheng Z, Zhou YZ, Zhao Y, Li JM, Zhou XM, Peng HL, Zhang GS, Liao XB and Fu XM: A novel STAT3 inhibitor attenuates angiotensin II-induced abdominal aortic aneurysm progression in mice through modulating vascular inflammation and autophagy. Cell Death Dis 11: 131, 2020

4. Li W, Xu C, Guo J, Liu K, Hu Y, Wu D, Fang H, Zou Y, Wei Z, Wang Z, et al: Cis- and Trans-Acting Expression Quantitative Trait Loci of Long Non-Coding RNA in 2,549 Cancers With Potential Clinical and Therapeutic Implications. Front Oncol 10 602104, 2020

5. Vishnoi A and Rani S: miRNA Biogenesis and Regulation of Diseases: An Overview. Methods Mol Biol 1509: 1-10, 2017.

6. Zhang S, Shen S, Yang Z, Kong X, Liu F and Zhen Z: Coding and Non-coding RNAs: Molecular Basis of Forest-Insect Outbreaks. Front Cell Dev Biol 8: 369, 2020.

7. Ala U: Competing Endogenous RNAs, Non-Coding RNAs and Diseases: An Intertwined Story. Cells 9: 9, 2020

8. Sun Y, Zhong L, He X, Wang S, Lai Y, Wu W, Song H, Chen Y, Yang Y, Liao W, et al: lncRNA H19 promotes vascular inflammation and abdominal aortic aneurysm formation by functioning as a competing endogenous RNA. J Mol Cell Cardiol 131: 66-81, 2019.

9. Lin H, You B, Lin X, Wang X, Zhou D, Chen Z, Chen Y and Wang R: Silencing of long non-coding RNA Sox2ot inhibits oxidative stress and inflammation of vascular smooth muscle cells in abdominal aortic aneurysm via microRNA-145-mediated Egr1 inhibition. Aging (Albany NY) 12: 12684-12702, 2020.

10. Tian Z, Sun Y, Sun X, Wang J and Jiang T: LINC00473 inhibits vascular smooth muscle cell viability to promote aneurysm formation via miR-212-5p/BASP1 axis. Eur J Pharmacol 873 : 172935, 2020.

11. Xia Q, Zhang L, Yan H, Yu L, Shan W and Jiang H: LUCAT1 contributes to MYRF-dependent smooth muscle cell apoptosis and may facilitate aneurysm formation via the sequestration of miR-199a-5p. Cell Biol Int 44: 755-763, 2020

12. Yang B, Wang X, Ying C, Peng F, Xu M, Chen F and Cai B: Long Noncoding RNA SNHG16 Facilitates Abdominal Aortic Aneurysm Progression through the miR-106b-5p/STAT3 Feedback Loop. J Atheroscler Thromb 28: 66-78, 2021.

13. Tian L, Hu X, He Y, Wu Z, Li D and Zhang H: Construction of lncRNA-miRNA-mRNA networks reveals functional lncRNAs in abdominal aortic aneurysm. Exp Ther Med 16: 3978-3986, 2018.

14. Han Y, Zhang H, Bian C, Chen C, Tu S, Guo J, Wu Y, Böckler D and Zhang J: Circular RNA Expression: Its Potential Regulation and Function in Abdominal Aortic Aneurysms. Oxid Med Cell Longev 2021: 9934951, 2021.

15. Zhao F, Chen T and Jiang N: CDR1as/miR-7/CKAP4 axis contributes to the pathogenesis of abdominal aortic aneurysm by regulating the proliferation and apoptosis of primary vascular smooth muscle cells. Exp Ther Med 19: 3760-3766, 2020.
16. Yue J, Zhu T, Yang J, Si Y, Xu X, Fang $\mathrm{Y}$ and $\mathrm{Fu} \mathrm{W}$ : circCBFB-mediated miR-28-5p facilitates abdominal aortic aneurysm via LYPD3 and GRIA4. Life Sci 253: 117533, 2020.

17. Lenk GM, Tromp G, Weinsheimer S, Gatalica Z, Berguer R and Kuivaniemi H: Whole genome expression profiling reveals a significant role for immune function in human abdominal aortic aneurysms. BMC Genomics 8: 237, 2007.

18. Biros E, Gäbel G, Moran CS, Schreurs C, Lindeman JH, Walker PJ, Nataatmadja M, West M, Holdt LM, Hinterseher I, et al: Differential gene expression in human abdominal aortic aneurysm and aortic occlusive disease. Oncotarget 6: 12984-12996, 2015.

19. Zhou M, Shi Z, Cai L, Li X, Ding Y, Xie T and Fu W: Circular RNA expression profile and its potential regulative role in human abdominal aortic aneurysm. BMC Cardiovasc Disord 20: 70, 2020.

20. Jolliffe IT and Cadima J: Principal component analysis: A review and recent developments. Philos Trans A Math Phys Eng Sci 374: 20150202, 2016.

21. RStudio Team: RStudio: Integrated Development for R. RStudio, Inc. Boston, MA, 2015. http://www.rstudio.com/. Accessed June 1, 2021

22. Huang W, Sherman BT and Lempicki RA: Systematic and integrative analysis of large gene lists using DAVID bioinformatics resources. Nat Protoc 4: 44-57, 2009.

23. Dudekula DB, Panda AC, Grammatikakis I, De S, Abdelmohsen K and Gorospe M: CircInteractome: A web tool for exploring circular RNAs and their interacting proteins and microRNAs. RNA Biol 13: 34-42, 2016

24. Sticht C, De La Torre C, Parveen A and Gretz N: miRWalk: An online resource for prediction of microRNA binding sites. PLoS One 13: e0206239, 2018

25. Agarwal V, Bell GW, Nam JW and Bartel DP: Predicting effective microRNA target sites in mammalian mRNAs. eLife 4: 4, 2015.

26. Kohl M, Wiese S and Warscheid B: Cytoscape: Software for visualization and analysis of biological networks. Methods Mol Biol 696: 291-303, 2011

27. Bader GD and Hogue CW: An automated method for finding molecular complexes in large protein interaction networks. BMC Bioinformatics 4: 2, 2003.

28. World Medical Association Declaration of Helsinki: Ethical principles for medical research involving human subjects. JAMA 310 : 2191-2194, 2013.

29. Livak KJ and Schmittgen TD: Analysis of relative gene expression data using real-time quantitative PCR and the 2(- $\Delta \Delta \mathrm{C}(\mathrm{T}))$ Method. Methods 25: 402-408, 2001.

30. Walter W, Sánchez-Cabo F and Ricote M: GOplot: An R package for visually combining expression data with functional analysis. Bioinformatics 31: 2912-2914, 2015.

31. Liu Y, Wang X, Wang $\mathrm{H}$ and $\mathrm{Hu} \mathrm{T}$ : Identification of key genes and pathways in abdominal aortic aneurysm by integrated bioinformatics analysis. J Int Med Res 48: 300060519894437, 2020.

32. Oh CK, Ko Y, Park JJ, Heo HJ, Kang J, Kwon EJ, Kang JW, Lee Y, Myung K, Kang JM, et al: FRZB as a key molecule in abdominal aortic aneurysm progression affecting vascular integrity. Biosci Rep 41: 41, 2021.

33. Luo Y and Huang C: CircSFMBT2 facilitates vascular smooth muscle cell proliferation by targeting miR-331-3p/HDAC5. Life Sci 264: 118691, 2021.

34. Guo X, Li D, Chen M, Chen L, Zhang B, Wu T and Guo R: miRNA-145 inhibits VSMC proliferation by targeting CD40. Sci Rep 6: 35302, 2016.

35. Liu R and Jin JP: Calponin isoforms CNN1, CNN2 and CNN3: Regulators for actin cytoskeleton functions in smooth muscle and non-muscle cells. Gene 585: 143-153, 2016.

36. Gong J, Zhou D, Jiang L, Qiu P, Milewicz DM, Chen YE and Yang B: In Vitro Lineage-Specific Differentiation of Vascular Smooth Muscle Cells in Response to SMAD3 Deficiency: Implications for SMAD3-Related Thoracic Aortic Aneurysm. Arterioscler Thromb Vasc Biol 40: 1651-1663, 2020.

37. Cheng Q, Li Z, Wang R, Zhang H, Cao H, Chen F, Li H, Xia Z, Feng S, Zhang H, et al: Genetic Profiles Related to Pathogenesis in Sporadic Intracranial Aneurysm Patients. World Neurosurg 131: e23-e31, 2019.

38. Feng HZ, Wang H, Takahashi K and Jin JP: Double deletion of calponin 1 and calponin 2 in mice decreases systemic blood pressure with blunted length-tension response of aortic smooth muscle. J Mol Cell Cardiol 129: 49-57, 2019.

39. Lesiak M, Augusciak-Duma A, Stepien KL, Fus-Kujawa A, Botor $\mathrm{M}$ and Sieron AL: Searching for new molecular markers for cells obtained from abdominal aortic aneurysm. J Appl Genet 62: 487-497, 2021. 
40. Ma K, Qiao Y, Wang H and Wang S: Comparative expression analysis of PD-1, PD-L1, and CD8A in lung adenocarcinoma. Ann Transl Med 8: 1478, 2020.

41. Sagan A, Mikolajczyk TP, Mrowiecki W, MacRitchie N, Daly K, Meldrum A, Migliarino S, Delles C, Urbanski K, Filip G, et al: T Cells Are Dominant Population in Human Abdominal Aortic Aneurysms and Their Infiltration in the Perivascular Tissue Correlates With Disease Severity. Front Immunol 10: 1979, 2019.

42. Li G, Zhou H, He Y, Sun S, Wu X and Yuan H: Ulinastatin Inhibits the Formation and Progression of Experimental Abdominal Aortic Aneurysms. J Vasc Res 57: 58-64, 2020.

43. Chen J, Lin M and Zhang S: Identification of key miRNA mRNA pairs in septic mice by bioinformatics analysis. Mol Med Rep 20: 3858-3866, 2019.

44. Xue Q, Wang X, Deng X, Huang Y and Tian W: CEMIP regulates the proliferation and migration of vascular smooth muscle cells in atherosclerosis through the WNT-beta-catenin signaling pathway. Biochem Cell Biol 98: 249-257, 2020.

45. Chen Y, Li L and Zhang J: Cell migration inducing hyaluronidase 1 (CEMIP) activates STAT3 pathway to facilitate cel proliferation and migration in breast cancer. J Recept Signal Transduct Res 41: 145-152, 2021.

46. Cui S and Zhang L: circ 001653 Silencing Promotes the Proliferation and ECM Synthesis of NPCs in IDD by Downregulating miR-486-3p-Mediated CEMIP. Mol Ther Nucleic Acids 20: 385-399, 2020.

47. Ito D and Kumanogoh A: The role of Sema4A in angiogenesis, immune responses, carcinogenesis, and retinal systems. Cell Adhes Migr 10: 692-699, 2016

48. Duijvis NW, Moerland PD, Kunne C, Slaman MMW, van Dooren FH, Vogels EW, de Jonge WJ, Meijer SL, Fluiter K and Te Velde AA: Inhibition of miR-142-5P ameliorates disease in mouse models of experimental colitis. PLoS One 12: e0185097, 2017.
49. Krishna SM, Seto SW, Jose RJ,Li J, Morton SK, Biros E, Wang Y, Nsengiyumva V, Lindeman JH, Loots GG, et al: Wnt Signaling Pathway Inhibitor Sclerostin Inhibits Angiotensin II-Induced Aortic Aneurysm and Atherosclerosis. Arterioscler Thromb Vasc Biol 37: 553-566, 2017.

50. Ma S, Wang DD, Ma CY and Zhang YD: MicroRNA-96 promotes osteoblast differentiation and bone formation in ankylosing spondylitis mice through activating the Wnt signaling pathway by binding to SOST. J Cell Biochem 120: 15429-15442, 2019.

51. Wei S, Zhang M, Zheng Y and Yan P: ZBTB16 Overexpression Enhances White Adipogenesis and Induces Brown-Like Adipocyte Formation of Bovine White Intramuscular Preadipocytes. Cell Physiol Biochem 48: 2528-2538, 2018.

52. Gerlinger-Romero F, Yonamine CY, Junior DC, Esteves JV and Machado UF: Dysregulation between TRIM63/FBXO32 expression and soleus muscle wasting in diabetic rats: Potential role of miR-1-3p, -29a/b-3p, and -133a/b-3p. Mol Cell Biochem 427: 187-199, 2017.

53. Dong Y and Simske JS: VertebrateClaudin/PMP22/EMP22/MP20 family protein TMEM47 regulates epithelial cell junction maturation and morphogenesis. Dev Dyn 245: 653-666, 2016.

(i)(9) This work is licensed under a Creative Commons Attribution-NonCommercial-NoDerivatives 4.0 International (CC BY-NC-ND 4.0) License. 